\title{
Risk factors of postoperative complications after emergency repair of incarcerated groin hernia for adult patients: a retrospective cohort study
}

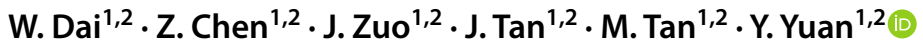

Received: 28 June 2018 / Accepted: 8 November 2018 / Published online: 12 November 2018

(c) The Author(s) 2018

\begin{abstract}
Purpose This study aimed to explore risk factors of postoperative complications for adult patients with incarcerated groin hernia (IGH).

Methods From January 2010 to December 2017, consecutive patients undergoing emergency hernia repair for IGH in our center were retrospectively reviewed. Postoperative complications, such as surgical site infection, seroma, hernia recurrence and mortality, were investigated, with risk factors for such complications analyzed using univariate and multivariate regressions.

Results Sixty-four patients were included, with 51 males and 13 females (mean age 65.1, range 25-98 years). Ten patients (15.6\%) underwent resection of necrotic bowel and anastomosis. 43 patients (67.2\%) received open tension-free herniorrhaphy with polypropylene mesh, whereas the rest (32.8\%) received herniorrhaphy without mesh. The overall postoperative complication rate was $40.6 \%$ (26/64), with an incisional complication rate of $31.2 \%(20 / 64)$ and an infection rate of $6.2 \%$ (4/64). At a median follow-up of 32 months, hernia recurrence and mortality were recorded in five cases each (7.8\%). Mesh repair was associated with decreased recurrence rate compared with non-mesh repair $(2.3 \%$ vs. 19.0\%, $p=0.019)$. Diabetes mellitus (OR 8.611, 95\%CI 1.292-57.405; $p=0.026$ ) was an independent risk factor of postoperative complications, together with chronic obstructive pulmonary disease (COPD; OR 14.365, 95\% CI 1.652-127.767, $p=0.016$ ), intestinal necrosis (OR $14.260,95 \%$ CI $1.079-188.460, p=0.044)$, and general anesthesia (OR 14.543, 95\%CI 1.682-125.711, $p=0.015$ ) as risk for incisional complications after surgery.

Conclusions Diabetes mellitus was an independent risk factor of postoperative complications for IGH, along with COPD, intestinal necrosis and general anesthesia associated with incisional complications. The use of polypropylene mesh did not increase infection or recurrence rate in this cohort.
\end{abstract}

Keywords Incarcerated groin hernia $\cdot$ Herniorrhaphy $\cdot$ Synthetic mesh $\cdot$ Complications $\cdot$ Risk factors

\section{Introduction}

W. Dai and Z. Chen equally contributed to this work.

M. Tan

tantommy@126.com

$\triangle$ Y. Yuan

condor.yyj@gmail.com

1 Center of Gastrointestinal Surgery, The First Affiliated Hospital of Sun Yat-sen University, Guangzhou 510080, Guangdong Province, People's Republic of China

2 Center of Hernia and Abdominal Wall Surgery, The First Affiliated Hospital of Sun Yat-sen University, Guangzhou 510080, Guangdong Province, People's Republic of China
Incarcerated abdominal wall hernia, defined as the inability to reduce the hernia content from abdominal wall, is a common surgical emergency, which accounts for 5-15\% of abdominal hernias. Incarcerated groin hernia (IGH) is one of the most frequently encountered types, accounting for $50-80 \%$ of incarcerated abdominal hernias. About $15 \%$ of IGH patients require bowel resection due to progressive bowel necrosis [1-4]. The incidence of postoperative complications in emergency repair of IGH is $21-39 \%$, along with a mortality rate of 4-5\% [1-4]. Early recognition of risk factors for postoperative complications and rapid effective 
interventions for potential complications are of great clinical significance.

The application of synthetic mesh in emergency surgery for IGH remains controversial, especially when the presence of bowel necrosis, contaminated or infected surgical field is clinically validated [5]. Recently, several studies have revealed that synthetic mesh could be safely and effectively used in patients with incarcerated or strangulated inguinal hernia, which significantly reduced the risk of hernia recurrence but did not increase the opportunity of surgical site infection (SSI) [1, 2, 4-8]. Nevertheless, most of those studies are retrospective analyses, and the level of evidencebased surgical practice is not high enough. As a result, more evidence-based clinical data are necessary to confirm the safety and effectiveness of using synthetic mesh in repairing IGH.

The purpose of this study was twofold: first, to investigate risk factors of postoperative complications for patients with IGH; and second, to evaluate the safety and efficacy of clinical usage of synthetic mesh in emergency herniorrhaphy for the IGH cohort.

\section{Methods}

From January 2010 to December 2017, consecutive adult patients who had confirmed IGH diagnosis and underwent emergency herniorrhaphy in our center were retrospectively reviewed. All data were retracted from medical records and our database of outpatient visits during the follow-up period. Patients who died from their comorbidities and those who were lost to follow-up were included for the final analysis. The study protocol was approved by the Institute Review Board of the ethical committee of our hospital, with written informed content waived due to its retrospective design.

The synthetic mesh used in emergency herniorrhaphy for all patients was polypropylene (PP) mesh, which was made of light-weight $\left(40 \mathrm{~g} / \mathrm{m}^{2}\right)$ and large-pore $(3 \mathrm{~mm})$ PP patch, and monofilament-braided wire $(0.13 \mathrm{~mm})$. This nonabsorbable mesh was commonly applied in open inguinal herniorrhaphy, with reduced incidence of chronic groin pain as well as other groin symptoms [9].

\section{Perioperative management}

Immediately after admission, antibiotic prophylaxis and fluid resuscitation were initiated against underlying infection and water-electrolyte imbalance. For patients with intestinal obstruction, nil per os and nasogastric tube decompression were kept before surgery. The emergency operation was basically decided by the main surgeons, according to their clinical judgment and preference. The administration of antibiotics would be extended for 5-7 days when bowel resection was performed due to confirmed necrosis, perforation, or severe surgical site contaminations. Additionally, surgical drainage and therapeutic antibiotic treatment according to bacteriologic culture results were continued for at least 7 days, when specific complications such as incisional and abdominal infection occurred.

During surgery, incarcerated hernia was repaired with various techniques. Briefly, as for IGH without ischemic necrosis of the hernia content, a simple reduction of the hernia content was applied, followed by a high ligation of hernia $\mathrm{sac}$ at the level of internal inguinal ring. After that, PP mesh was used to achieve tension-free repair when the Lichtenstein procedure was considered for inguinal hernia, with non-mesh tissue repair performed when Bassini or Shouldice procedure was considered [10]. The mesh-plug repair was employed for femoral hernia, with non-mesh tissue repair performed when the McVay procedure was preferred [11].

As for IGH with bowel necrosis but no perforation, a resection of necrotic bowel plus extracorporeal anastomosis via an inguinal incision was performed, as illustrated in Fig. 1. Afterward, a high ligation of hernia sac was performed, followed by a mesh or tissue repair mainly determined by surgeons. As for IGH with bowel perforation, a laparotomy from a mid-line incision would be made, followed by a simultaneous bowel resection and a high ligation of hernia sac.

A closed non-suction drainage system was supplied to the surgical wound once an enterectomy performed or contaminated wound confirmed during surgery. Of note, the end of inserted drain should be placed into peritoneal cavity in specific cases with bowel necrosis or peritonitis. The drain was removed after the drainage had decreased to $50 \mathrm{ml} /$ day.

After discharge, patients were scheduled to regular outpatient visits at least for a 1-year follow-up period. During these visits, physical examination, abdominal ultrasound, particularly CT scans were performed to determine hernia recurrence and other long-term complications. The end date of follow-up was March 31, 2018. A recorded death or recurrence was regarded as the endpoint of the study.

\section{Data collection and study outcomes}

All included cases were reviewed by two researchers (DW and CZ) independently. Clinical data, including demographic characteristics (gender, age, concomitant disease, etc.) and basic information of IGH, were collected from the medical records and database meanwhile. Next, data related to postoperative complications were extracted and double checked by both researchers. Surgical wound classification was consistent with the Centers for Disease Control and Prevention (CDC) wound classification, as well as the diagnostic criteria of SSI [12]. 
Fig. 1 Demonstration of surgical management of incarcerated groin hernia. This was a 46-year-old male patient who presented with severe groin pain and abdominal distension for $10 \mathrm{~h}$. a Coronal view of abdominal CT scan; b sagittal view of abdominal ct scan; $\mathbf{c}$ surgical exploration from the groin incision; $\mathbf{d}$ resected bowel and greater omentum strangulated in the right groin area. The white arrows in both $\mathbf{a}$ and $\mathbf{b}$ show the strangulated inguinal hernia on the right-sided groin. In addition, the patient has intestinal obstruction. White arrow heads (c and $\mathbf{d})$ refer to intestinal necrosis
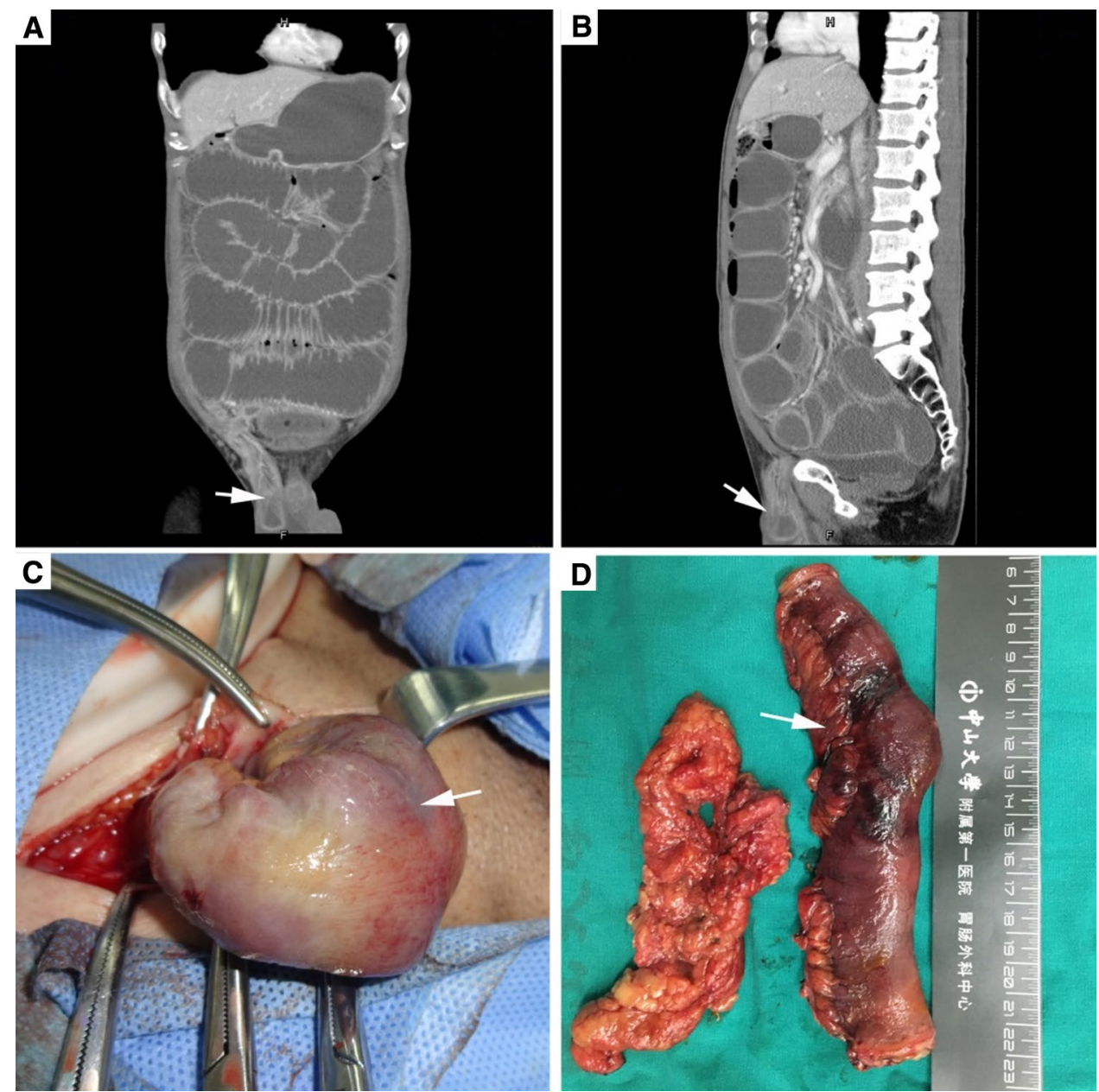

The primary outcomes included postoperative complications within 30 days of surgery, hernia recurrence and long-term mortality during the follow-up period. Patients were then stratified into two groups based on mesh or non-mesh repair technique applied during surgery to evaluate the safety and efficacy of treatment options.

\section{Statistical analysis}

Descriptive data were presented as mean \pm standard deviation (SD) or median (range or 95\% confidential interval $[95 \% \mathrm{CI}])$ as appropriate. Chi-square test was used for the comparison of categorical variables. Student's $t$ test and Mann-Whitney $U$ test were employed for comparisons of normal and non-normal continuous variables, respectively. Univariate and multivariate logistic regressions were used to explore risk factors of postoperative complications. All data analyses were performed using the SPSS for windows (version 23, IBM, Chicago, IL,
USA). A two-tailed $p$ value $<0.05$ or an odds ratio (OR) with $95 \% \mathrm{CI}$ not inclusive of the unity was considered significant.

\section{Results}

Within the study period, a total of 64 patients were included, with 51 males (79.7\%) and 13 females (20.3\%), and an average age of 65.1 (range 25-98) years. The baseline characteristics of all subjects are summarized in Table 1. It showed that male was more involved than female, almost reaching up to $80 \%$ of cases. Besides, $57.8 \%$ of all patients were older than 65 years, and $62.5 \%$ of them had various concomitant diseases when admitting to our center.

The median duration of groin hernia for this cohort was 60 (range 1-70) months. The median time interval until diagnosis of IGH was 24 (range 2-168) hours. The median size of hernia sac was 7 (range $3-18$ ) $\mathrm{cm}$ in diameter. 
Table 1 Baseline characteristics of patients with incarcerated groin hernia

\begin{tabular}{|c|c|c|c|c|}
\hline Variables & The pooled $(N=64)$ & Mesh group $(N=43)$ & Non-mesh group $(N=21)$ & $p$ value \\
\hline Gender (male), $n(\%)$ & $51(79.7)$ & $36(83.7)$ & $15(71.4)$ & 0.324 \\
\hline Age (years), mean \pm SD & $65.1 \pm 17.1$ & $62.5 \pm 17.9$ & $70.5 \pm 14.1$ & 0.078 \\
\hline$\geq 65$ years, $n(\%)$ & $37(57.8)$ & $21(48.8)$ & $16(76.2)$ & 0.058 \\
\hline Concomitant disease conditions, $n(\%)$ & $40(62.5)$ & $23(53.5)$ & $17(81.0)$ & 0.053 \\
\hline Diabetes mellitus & $12(18.8)$ & $6(14.0)$ & $6(28.6)$ & 0.185 \\
\hline Hypertension & $33(51.6)$ & $18(41.9)$ & $15(71.4)$ & $0.035 *$ \\
\hline Heart diseases & $15(23.4)$ & $9(20.9)$ & $6(28.6)$ & 0.540 \\
\hline COPD & $14(21.9)$ & $7(16.3)$ & $7(33.3)$ & 0.196 \\
\hline Chronic nephropathy/renal failure & $2(3.1)$ & $1(2.3)$ & $1(4.8)$ & 1.000 \\
\hline Duration of hernia (years), $M(95 \% \mathrm{CI})$ & $5.0(5.4-12.0)$ & $5(3.4-6.0)$ & $7(2.3-10.0)$ & 0.891 \\
\hline Duration of hernia incarceration (h), $M(95 \% \mathrm{CI})$ & $24.0(32.4-52.5)$ & $23(13.0-48.0)$ & $25(14.7-48.0)$ & 0.365 \\
\hline Maximum diameter of hernia sac $(\mathrm{cm}), M(95 \% \mathrm{CI})$ & $7(6.9-8.8)$ & $6(5.8-8.0)$ & $8(5.8-13.3)$ & 0.117 \\
\hline Bowel necrosis, $n(\%)$ & $10(15.6)$ & $1(2.3)$ & $9(42.9)$ & $<0.001^{*}$ \\
\hline Bowel perforation, $n(\%)$ & $2(3.1)$ & 0 & $2(9.5)$ & 0.104 \\
\hline Preoperative intestinal obstruction, $n(\%)$ & $22(34.4)$ & $9(20.9)$ & $13(61.9)$ & $0.002 *$ \\
\hline Abdominal wall hernia history, $n(\%)$ & $4(6.2)$ & & & \\
\hline Abdominal surgery history, $n(\%)$ & $6(9.4)$ & $4(9.3)$ & $2(9.5)$ & 1.000 \\
\hline Classification of groin hernia, $n(\%)$ & & & & $0.020 *$ \\
\hline Indirect hernia & $47(73.4)$ & $34(79.1)$ & $13(61.9)$ & \\
\hline Direct hernia & $5(7.8)$ & $5(11.6)$ & 0 & \\
\hline Femoral hernia & $11(17.2)$ & $4(9.3)$ & $7(33.3)$ & \\
\hline Saddle-bag hernia & $1(1.6)$ & 0 & $1(4.8)$ & \\
\hline Recurrent hernia, $n(\%)$ & $3(4.7)$ & $3(7.0)$ & 0 & 0.545 \\
\hline Scrotal hernia, $n(\%)$ & $43(84.3)$ & $30(83.3)$ & $13(86.7)$ & 1.000 \\
\hline Gilbert-Rutkow type, $n(\%)$ & & & & $0.039 *$ \\
\hline II & $5(7.8)$ & $5(11.6)$ & 0 & \\
\hline III & $42(65.6)$ & $29(67.4)$ & $13(61.9)$ & \\
\hline VI & $4(6.2)$ & $4(9.3)$ & 0 & \\
\hline $\mathrm{V}$ & $1(1.6)$ & $1(2.3)$ & 0 & \\
\hline VI & $1(1.6)$ & 0 & $1(4.8)$ & \\
\hline VII & $11(17.2)$ & $4(9.3)$ & $7(33.3)$ & \\
\hline Hernia location (right sided), $n(\%)$ & $40(62.5)$ & $23(53.5)$ & 17 (81.0) & 0.053 \\
\hline
\end{tabular}

$S D$ standard deviation, $M$ median, $C I$ confidence interval, $C O P D$ chronic obstructive pulmonary disease

Scrotal hernia defined as hernia extending into the scrotum, Saddle-bag hernia defined as a combination of indirect and direct sacs on both sides of the inferior epigastric vessels, *Statistically significant

Bowel necrosis was recorded in 10 cases $(15.6 \%)$, with intestinal perforation in 2 cases $(3.1 \%)$ and intestinal obstruction in 22 cases (34.4\%). IGH occurred more frequently in the right-sided groin than in the left-sided groin [62.5\% (40/64) vs. $37.5 \%$ (24/64)], and the rate of bowel necrosis in femoral hernia was significantly higher than that in inguinal hernia $[36.3 \%(4 / 11)$ vs. $11.3 \%(6 / 53)$, $p=0.037]$.

\section{Surgical data}

Emergency herniorrhaphy was successfully completed in all patients, with no postoperative death or readmission observed until 30 days after discharge. Open tension-free herniorrhaphy was performed in 43 cases (67.2\%), with non-mesh herniorrhaphy applied in the rest of 21 cases (32.8\%). Laparoscopic herniorrhaphy was not recorded. The detailed surgical information is shown in Table 2. Briefly, the median operation time was 95 (range 50-180) min. Bowel resection and anastomosis were performed in ten cases (15.6\%). Among those ten patients, one patient received PP mesh repair after a resection of necrotic bowel since the surgical field was presumed clean. The remaining nine patients underwent non-mesh tissue repair alone after bowel resection. 
Table 2 Perioperative data of patients with incarcerated groin hernia

\begin{tabular}{lc}
\hline Variables & Value \\
\hline ASA grades, $n(\%)$ & \\
Grade I & $23(35.9)$ \\
Grade II & $28(43.8)$ \\
Grade III & $13(20.3)$ \\
Anesthetic methods, $n(\%)$ & \\
Local & $4(6.2)$ \\
Spinal & $32(50.0)$ \\
General & $28(43.8)$ \\
Operation time (min), M (95\%CI) & $95(91.9-107.8)$ \\
Open tension-free herniorrhaphy, $n(\%)$ & $43(67.2)$ \\
Lichtenstein & $26(40.6)$ \\
Mesh-plug repair & $10(15.6)$ \\
Preperitoneal herniorrhaphy & $7(10.9)$ \\
Traditional herniorrhaphy, $n(\%)$ & $21(32.8)$ \\
High ligation of hernia sac & $4(6.2)$ \\
Laparotomy plus high ligation & $2(3.1)$ \\
Bassini method & $8(1.25)$ \\
McVay method & $6(9.37)$ \\
Shouldice method & $1(1.56)$ \\
Postoperative LOS (days), $M(95 \%)$ & $5.0(5.3-7.9)$ \\
Enterectomy, $n$ (\%) & $10(15.6)$ \\
Small intestine & $9(14.06)$ \\
Caecum & $1(1.56)$ \\
\hline
\end{tabular}

$M$ median, $C I$ confidence interval, ASA American society of anesthesia score, LOS length of stay

Table 3 Postoperative complications and follow-up results

\begin{tabular}{lc}
\hline Variables & Value \\
\hline Postoperative complications, $n(\%)$ & $26(40.6)$ \\
Incisional complications & $20(31.2)$ \\
Seroma & $19(29.7)$ \\
Incisional infection & $4(6.2)$ \\
Scrotal swelling & $13(27.1)$ \\
Retention of urine & $6(9.4)$ \\
Intra-abdominal infection & $1(1.5)$ \\
Acute heart failure/arrhythmia & $2(3.1)$ \\
Pulmonary infection & $1(1.5)$ \\
Mesh infection & $1(2.3 \%)$ \\
Mesh extraction & $0(0)$ \\
Postoperative follow-up duration (months), $M$ & $32(31.6-43.8)$ \\
(95\%CI) & $57(89.1)$ \\
Access to follow-up cases, $n(\%)$ & $5(7.8)$ \\
Hernia recurrence, $n(\%)$ & $5(7.8)$ \\
Hernia unrelated death, $n(\%)$ & \\
\hline
\end{tabular}

$M$ median, $C I$ confidence interval

\section{Postoperative complications and long-term outcomes}

The overall postoperative complication rate was $40.6 \%$ (26/64), and the incisional complication rate was $31.2 \%$ (20/64). Local wound complications were much more commonly observed than other complications (Table 3). Of note, one case repaired with PP mesh developed mild mesh infection after 6 days of surgery; however, the mesh was not retrieved from the surgical site after an effective wound healing process using a negative pressure drainage system. No additional surgery was appended for those complications according to data collected from medical records.

At the end time of follow-up, 57 of the 64 patients $(89.1 \%)$ had complete follow-up records, with a median follow-up period of 32 (range, 31.6-43.8) months. Briefly, two patients $(3.1 \%)$ lost contact with us, and five patients (7.8\%) died. Of the five dead cases, two died of heart disease, two of multiple organ failure and one of respiratory failure. Hernia-related death was not observed, but hernia recurrence after initial herniorrhaphy was recorded in five cases $(7.8 \%)$. All relapsed hernias were successfully managed with second operations.

\section{Mesh versus non-mesh repair}

To evaluate the safety and efficacy of mesh repair for IGH treatment, all patients were divided into two groups: 43 cases $(67.2 \%)$ and 21 cases $(32.8 \%)$ in the mesh and non-mesh repair groups, respectively (Table 4 ). The average operation time in the mesh group was much shorter than that in the non-mesh group (87.4 min vs. $125.5 \mathrm{~min}, p<0.001)$. Importantly, the incidence of overall postoperative complication $(27.9 \%$ vs. $66.7 \%, p=0.005)$, incisional seroma (20.9\% vs. $47.6 \%, p=0.028)$ and scrotal swelling $(13.9 \%$ vs. $66.7 \%, p=0.001)$, and median postoperative length of stay ( 5 vs. 8 days, $p=0.026$ ) were markedly decreased in the mesh repair group compared with the non-mesh repair group. Besides, the hernia recurrence rate was also significantly reduced $(2.3 \%$ vs. $19.1 \%, p=0.019)$, with a comparable mortality rate observed between both groups $(7.0 \%$ vs. $9.5 \%, p=0.721)$.

\section{Risk factors of postoperative complications}

The univariate regression analysis indicated that concomitant basic disease $(p=0.049)$, diabetes mellitus (DM, $p=0.001$ ), chronic heart disease (CHD, $p=0.019$ ), history of abdominal wall hernia $(p=0.024)$, bowel necrosis $(p=0.011)$, general anesthesia $(p=0.009)$, and mesh repair $(p=0.003)$ were risk factors of postoperative complications for IGH patients (Table 5). Specifically, concomitant 
Table 4 Comparisons of perioperative data between mesh repair group and nonmesh repair group

\begin{tabular}{llll}
\hline Variables & Mesh repair group $(n=43)$ & $\begin{array}{l}\text { Non-mesh repair } \\
\text { group }(n=21)\end{array}$ & $p$ value \\
\hline Anesthetic methods, $n(\%)$ & & & $0.001^{*}$ \\
Local & $3(7.0)$ & $1(4.8)$ & \\
Spinal & $28(65.1)$ & $4(19.0)$ & \\
General & $12(27.9)$ & $16(76.2)$ & \\
Operation time (min), mean $\pm \mathrm{SD}$ & $87.4 \pm 17.8$ & $125.5 \pm 38.5$ & $<0.001^{*}$ \\
Enterectomy, $n(\%)$ & $1(2.3)$ & $9(42.9)$ & $<0.001^{*}$ \\
Postoperative complications, $n(\%)$ & $12(27.9)$ & $14(66.7)$ & $0.003^{*}$ \\
Incisional seroma & $9(20.9)$ & $10(47.6)$ & $0.028^{*}$ \\
Incisional infection & $1(2.3)$ & $3(14.3)$ & 0.099 \\
Scrotal swelling & $5(11.6)$ & $8(38.1)$ & $0.001^{*}$ \\
Mesh infection & $1(2.3)$ & 0 & $\mathrm{NA}$ \\
Urine retention & $3(7.0)$ & $3(14.3)$ & 0.385 \\
Postoperative LOS (days), $M(95 \% \mathrm{CI})$ & $5(3.8-6.0)$ & $8(5.0-8.0)$ & $0.026^{*}$ \\
Follow-up period (months), $M(95 \% \mathrm{CI})$ & $32(24.5-46.8)$ & $32(22.4-53.2)$ & 0.672 \\
Recurrence rate, \% (n/N) & $2.3(1 / 43)$ & $19.0(4 / 21)$ & $0.019^{*}$ \\
Overall mortality rate, \% (n/N) & $7.0(3 / 43)$ & $9.5(2 / 21)$ & 0.721 \\
\hline
\end{tabular}

$N A$ not available, $M$ median value, $C I$ confidential interval, $S D$ standard deviation, $L O S$ length of stay, $N$ total number

*Statistically significant basic disease $(p=0.002), \mathrm{DM}(p<0.001)$, hypertension $(p=0.047)$, CHD $(p=0.010)$, chronic obstructive pulmonary disease (COPD, $p=0.001)$, bowel necrosis $(p=0.001)$, general anesthesia $(p=0.016)$, and mesh repair $(p=0.028)$ were risk factors for postoperative incisional complications.

The stepwise multivariate logistic analysis is summarized in Fig. 2. It showed that DM ( $p=0.026)$ was an independent risk factor of postoperative complications, with DM ( $p=0.011)$, COPD $(p=0.016)$, intestinal necrosis $(p=0.044)$ and general anesthesia $(p=0.015)$ as independent risk for incisional complications. Importantly, mesh repair was not independently associated with those complications for IGH.

\section{Discussion}

In this study, the overall postoperative complication rate was $40.6 \%$, with $31.2 \%$ and $6.2 \%$ for incisional complication rate and wound infection rate, respectively. Within the follow-up period, hernia recurrence and death were recorded in $7.8 \%$ of included patients each. Importantly, tension-free herniorrhaphy with PP mesh was performed in $67.2 \%$ of included subjects, and it was insignificantly associated with postoperative complications.

IGH in adult often involves bowel obstruction and potential necrosis, and it needs an emergency surgery to avoid death-related complications [13-15]. It is known that most of IGHs have the following characteristics: elder age, weakness due to concomitant disease, a long history of hernia with a large sac, and delayed diagnosis and treatment. Those features often lead to bowel necrosis or perforation, even severe complications such as diffuse peritonitis and sepsis, which are associated with increased rates of postoperative morbidity and mortality [3, 4, 16, 17]. A previous retrospective study indicates that long history of hernia, prolonged length of stay, severe concomitant diseases and high American Society of Anesthesia (ASA) grade were significant factors linked to unfavorable outcome of IGH [18].

Many studies have shown that the presence of concomitant diseases, such as DM, cardiovascular disorders, COPD and kidney disease, prolonged the length of stay and increased morbidity and mortality in IGH patients [2-4, 8, 16-19]. It is speculated that significant concomitant diseases greatly depressed oxygenation of surgical field, subsequently attenuated wound healing process and increased risk of wound complications after surgery [20, 21]. Additionally, numerous studies have confirmed that the presence of bowel necrosis or perforation made IGH patients more prone to bacterial translocation and wound infection after surgery [8, 10, 18, 19, 22-24]. In our study, DM was found to be an independent risk factor of postoperative complications, together with COPD, bowel necrosis and general anesthesia as independent risk factors for incisional complications. Our findings were in accordance with other studies [1, 6, 15-18, 22, 24].

Previous studies have reported that general or spinal anesthesia could increase the incidence of postoperative 
Table 5 Univariate regression analysis of risk factors of postoperative complications for incarcerated groin hernia

\begin{tabular}{|c|c|c|c|c|c|c|}
\hline \multirow[t]{2}{*}{ Variables } & \multicolumn{3}{|l|}{ Postoperative complications } & \multicolumn{3}{|l|}{ Incisional complications } \\
\hline & Control $(N=38)$ & Event $(N=26)$ & $p$ value & Control $(N=44)$ & Event $(N=20)$ & $p$ value \\
\hline $\begin{array}{l}\text { Gender (male/female), } \\
n(\%)\end{array}$ & $31(81.6) / 7(18.4)$ & $20(76.9) / 6(23.1)$ & 0.649 & $38(86.4) / 6(13.6)$ & $13(65.0) / 7(35.0)$ & 0.090 \\
\hline Age ( $\geq 65$ years), $n(\%)$ & $19(50.0)$ & $18(69.2)$ & 0.126 & $22(50.0)$ & $15(75.0)$ & 0.061 \\
\hline $\begin{array}{l}\text { Concomitant basic dis- } \\
\text { ease, } n(\%)\end{array}$ & $20(52.6)$ & $20(76.9)$ & $0.049 *$ & $22(50.0)$ & $18(90.0)$ & $0.002 *$ \\
\hline Diabetes mellitus & $2(5.3)$ & $10(38.5)$ & $0.001 *$ & $2(4.6)$ & $10(50.0)$ & $<0.001^{*}$ \\
\hline Hypertension & $17(44.7)$ & $16(61.5)$ & 0.187 & $19(43.2)$ & $14(70.0)$ & $0.047 *$ \\
\hline Heart disease & $5(13.2)$ & $10(38.5)$ & $0.019^{*}$ & $6(13.6)$ & $9(45.0)$ & $0.010^{*}$ \\
\hline COPD & $6(15.8)$ & $8(30.8)$ & 0.155 & $4(9.1)$ & $10(50.0)$ & $0.001 *$ \\
\hline Chronic kidney disease & $1(2.6)$ & $1(3.8)$ & 1.000 & $2(4.6)$ & 0 & 1.000 \\
\hline Scrotal hernia, $n(\%)$ & $26(68.4)$ & $17(65.4)$ & 1.000 & $30(68.2)$ & $13(65.0)$ & 0.419 \\
\hline $\begin{array}{l}\text { Duration of hernia (years), } \\
\qquad M(95 \% \mathrm{CI})\end{array}$ & $5(3.0-6.5)$ & $5.5(2.0-10.0)$ & 0.431 & $5(4.7-7.5)$ & $4(1.8-10.0)$ & 0.599 \\
\hline Duration $\geq 5$ years, $n(\%)$ & $23(60.5)$ & $15(57.7)$ & 0.821 & $28(63.6)$ & $10(50.0)$ & 0.303 \\
\hline $\begin{array}{l}\text { Duration of incarceration } \\
\text { (h), } M(95 \% \mathrm{CI})\end{array}$ & $23(12.0-48.0)$ & $36(18.0-80.0)$ & 0.115 & $23(18.0-48.0)$ & $23.5(12.0-63.7)$ & 0.738 \\
\hline Duration $\geq 24 \mathrm{~h}, n(\%)$ & $17(44.7)$ & $15(57.7)$ & 0.309 & $21(47.7)$ & $11(55.0)$ & 0.590 \\
\hline $\begin{array}{l}\text { History of abdominal wall } \\
\text { hernia, } n(\%)\end{array}$ & 0 & $4(15.4)$ & $0.024 *$ & $2(4.6)$ & $2(10.0)$ & 0.583 \\
\hline $\begin{array}{l}\text { History of abdominal } \\
\text { surgery, } n(\%)\end{array}$ & $3(7.9)$ & $3(11.5)$ & 0.680 & $3(6.8)$ & $3(15.0)$ & 0.366 \\
\hline $\begin{array}{l}\text { Maximum diameter of } \\
\text { hernia sac }(\mathrm{cm}), M \\
(95 \% \mathrm{CI})\end{array}$ & $6(5.0-8.0)$ & $8(5.0-10.0)$ & 0.123 & $6.5(5.0-8.0)$ & $9(5.0-12.0)$ & 0.161 \\
\hline Diameter $\geq 10 \mathrm{~cm}, n(\%)$ & $10(26.3)$ & $12(46.1)$ & 0.101 & $12(27.3)$ & $10(50.0)$ & 0.076 \\
\hline Intestinal necrosis, $n(\%)$ & $2(5.3)$ & $8(30.8)$ & $0.011^{*}$ & $2(4.6)$ & $8(40.0)$ & $0.001 *$ \\
\hline $\begin{array}{l}\text { Intestinal perforation, } \\
n(\%)\end{array}$ & 0 & $2(7.7)$ & 0.161 & 0 & $2(10.0)$ & 0.094 \\
\hline $\begin{array}{l}\text { Concomitant intestinal } \\
\text { obstruction, } n(\%)\end{array}$ & $11(28.9)$ & $11(42.3)$ & 0.269 & $13(29.6)$ & $9(45.0)$ & 0.228 \\
\hline $\begin{array}{l}\text { Classification of hernia, } \\
n(\%)\end{array}$ & & & 0.476 & & & 0.283 \\
\hline Indirect inguinal hernia & $27(71.0)$ & $20(76.9)$ & & $34(77.3)$ & $13(65.0)$ & \\
\hline Direct hernia & $4(10.5)$ & $1(3.8)$ & & $4(9.1)$ & $1(5.0)$ & \\
\hline Femoral hernia & $7(18.4)$ & $4(15.4)$ & & $6(13.6)$ & $5(25.0)$ & \\
\hline Saddle-bag hernia & 0 & $4(15.4)$ & & 0 & $1(5.0)$ & \\
\hline Recurrent hernia, $n(\%)$ & $1(2.6)$ & $2(7.7)$ & 0.561 & $1(2.3)$ & $2(10.0)$ & 0.228 \\
\hline $\begin{array}{l}\text { Hernia location (right- } \\
\text { sided), } n(\%)\end{array}$ & $23(60.5)$ & $17(65.4)$ & 0.693 & $27(61.4)$ & $13(65.0)$ & 0.781 \\
\hline $\begin{array}{l}\text { ASA grades (I/II/III), } \\
n(\%)\end{array}$ & $\begin{array}{l}16(42.1) / 17(44.7) / 5 \\
\quad(13.2)\end{array}$ & $7(26.9) / 11(42.3) / 8(30.8)$ & 0.186 & $\begin{array}{l}20(45.5) / 17(38.6) / 7 \\
\quad(15.9)\end{array}$ & $3(15.0) / 11(55.0) / 6(30.0)$ & 0.057 \\
\hline $\begin{array}{l}\text { Anesthetic methods (local/ } \\
\text { spinal/general), } n(\%)\end{array}$ & $2(5.3) / 25(65.8) / 11(28.9)$ & $2(7.7) / 7(26.9) / 17(65.4)$ & $0.009^{*}$ & $3(6.8) / 27(61.4) / 14(31.8)$ & $1(5.0) / 5(25.0) / 14(70.0)$ & $0.016^{*}$ \\
\hline Mesh repair (yes), $n(\%)$ & 31 (81.6) & $12(46.2)$ & $0.003^{*}$ & $11(25.0)$ & $10(50.0)$ & $0.028 *$ \\
\hline $\begin{array}{l}\text { Operation time }(\mathrm{min}), \\
\text { mean } \pm \mathrm{SD}\end{array}$ & $96.3 \pm 26.3$ & $105.1 \pm 36.1$ & 0.300 & $92.9 \pm 24.3$ & $115.2 \pm 40.5$ & 0.053 \\
\hline
\end{tabular}

$M$ median value, $C I$ confidential intervals, $S D$ standard deviation, $C O P D$ chronic obstructive pulmonary disease, $A S A$ American society of anesthesia score

*Statistically significant

complications compared to local anesthesia $[3,8]$. It is believed that local anesthesia has fewer adverse effects on respiratory function and provides better postoperative pain control in comparison with general anesthesia [25]. In clinical practice, selection of anesthetic technique is mainly determined by the severity of ASA class and intestinal incarceration. Consequently, general anesthesia was more 
Fig. 2 Multivariate logistic analysis of postoperative complications for incarcerated groin hernia. a Risk factors of postoperative complications; $\mathbf{b}$ risk factors of incisional complications after surgery. Factors that were statistically significant in univariate regression analysis were included in this stepwise logistic analysis. HTN, hypertension; COPD, chronic obstructive pulmonary disease; $\mathrm{OR}$, odds ratio; $\mathrm{CI}$, confidential interval. $P<0.05$ denotes an independent risk factor of outcomes

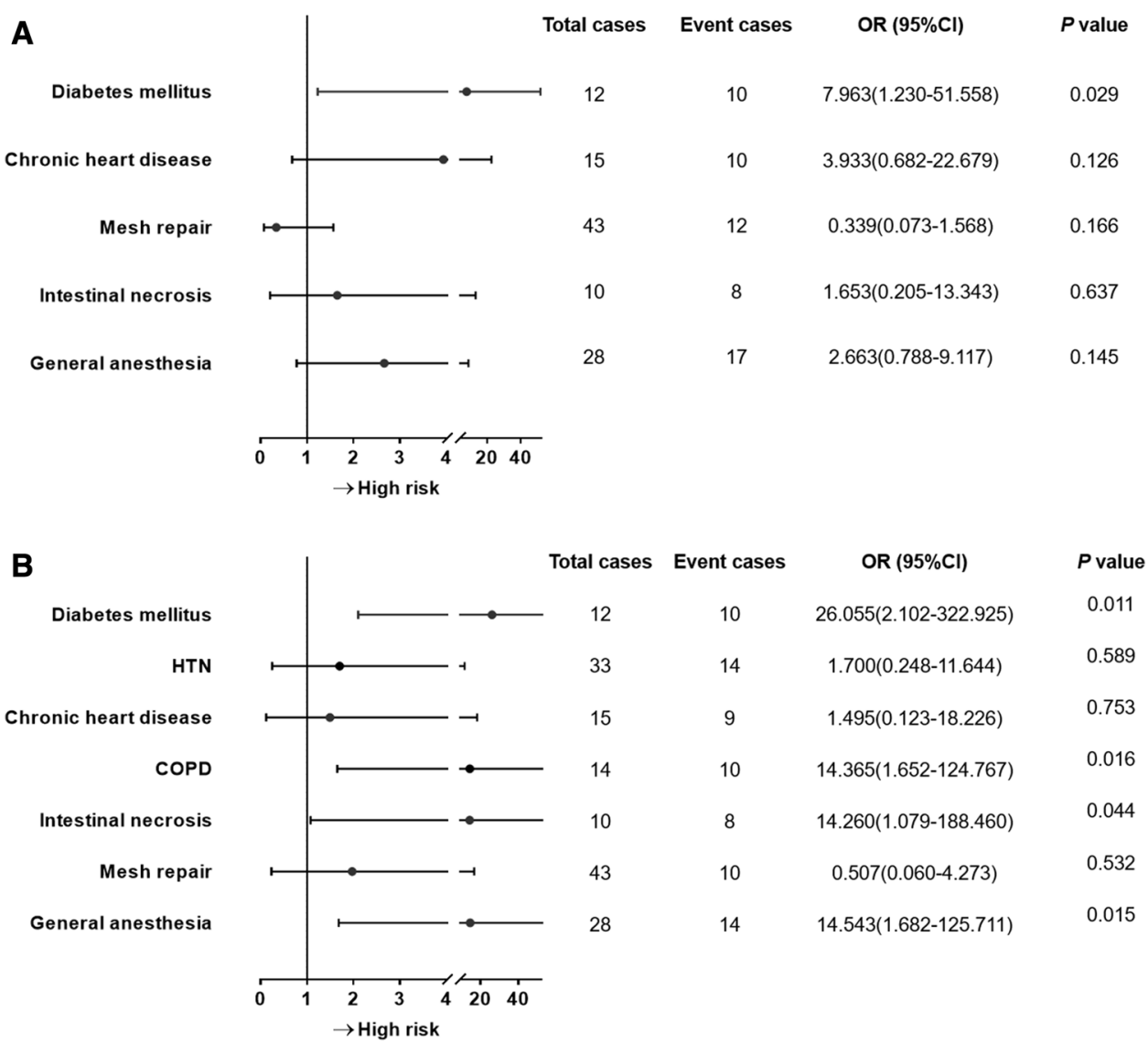

predicated on patient selection bias by surgeons, with further studies required to obtain more powerful evidence-based data. Clinical utility of PP mesh can be considered when a hernia sac has been properly managed without residual contamination, and tension-free herniorrhaphy with such mesh has been confirmed to reduce recurrence and SSI rates [5, $10,31]$.

As is well known, enterectomy is one of the worst complications of IGH, remaining an important risk factor of postoperative morbidity and mortality $[4,5,10,24]$. It is reported that incarceration greater than $24 \mathrm{~h}$, bowel perforation, severe hernia sac contamination and generalized peritonitis almost lead to an unavoidable enterectomy and make synthetic mesh repair unpracticable [22]. Under those conditions, use of biological mesh would be an alternative option, and several studies have shown its efficacy on potentially contaminated or infected herniorrhaphy [32].

This study had several limitations. First, the surgical strategy was made by surgeons' preference, which came with an unavoidable selection bias. Tension-free herniorrhaphy was commonly selected for younger patients with shorter hernia duration and lower ASA grade. However, non-mesh tissue repair was applied for older patients with longer course, more significant concomitant illnesses, and bowel obstruction or necrosis. Besides, anesthetic regimen was often determined by 
the ASA class and personal experience of on-call anesthesiologists during surgery. Hence, general anesthesia was frequently applied in this cohort. Second, several confounding factors such as subjective factors of on-call surgeons and concrete concomitant diseases of these patients were hard to balance due to the small sample size of our cohort. At last, chronic pain degree and life of quality after surgery were not evaluated here due to its retrospective design. Other future studies with larger sample size and prospective study design must be required to illustrate the current findings.

\section{Conclusion}

This study confirmed that DM was an independent risk factor of postoperative complications after emergency surgery for IGH, alongside with DM, COPD, intestinal necrosis and general anesthesia considered as risk factors for specific incisional complications. Mesh repair with various tension-free techniques could be safely and effectively performed for IGH patients, with better long-term outcomes obtained compared with non-mesh tissue repair.

Acknowledgements The authors Ms. Z for her nice works in routine telephone interview of patients after surgery. We are also very grateful to $\mathrm{Ms} . \mathrm{N}$ for helping with the manuscript.

Author Contributions Study conception and design: TM and YY; obtained permission for IRB: YY. Acquisition of data: CZ, ZJ, DW and TJ; analysis and interpretation of data: YY, TM and TJ. Preparation of study tools: DW, TJ, ZJ; drafting of manuscript: YY, CZ and DW; critical revision: $\mathrm{YY}$ and TM.

Funding This work was supported by grants from National Natural Science Foundation of China (No. 81401307) and Guangdong Province science and technology planning project (2016A0103038).

\section{Compliance with ethical standards}

Conflict of interest Dr. DW declares no conflict of interest related to the submitted work. Dr. CZ declares no conflict of interest related to the submitted work. Dr. ZJ declares no conflict of interest related to the submitted work. Dr. TJ declares no conflict of interest related to the submitted work. Dr. TM declares no conflict of interest related to the submitted work. Dr. YY declares no conflict of interest related to the submitted work.

Ethical approval Ethical approval was agreed by the ethical committee of our institution, Sun Yat-sen University First Affiliated Hospital, Guangzhou, People's Republic of China.

Human and animal rights All procedures performed in studies involving human participants were in accordance with the ethical standards of the institutional and/or national research committee and with the 1964 Helsinki declaration and its later amendments or comparable ethical standards.

Informed consent Informed consent was waived from all participants.
Open Access This article is distributed under the terms of the Creative Commons Attribution 4.0 International License (http://creativeco mmons.org/licenses/by/4.0/), which permits unrestricted use, distribution, and reproduction in any medium, provided you give appropriate credit to the original author(s) and the source, provide a link to the Creative Commons license, and indicate if changes were made.

\section{References}

1. Bessa SS, Abdel-fattah MR, Al-Sayes IA, Korayem IT (2015) Results of prosthetic mesh repair in the emergency management of the acutely incarcerated and/or strangulated groin hernias: a 10-year study. Hernia 19:909-914

2. Venara A, Hubner M, Le Naoures P et al (2014) Surgery for incarcerated hernia: short-term outcome with or without mesh. Langenbecks Arch Surg 399:571-577

3. Derici H, Unalp HR, Bozdag AD et al (2007) Factors affecting morbidity and mortality in incarcerated abdominal wall hernias. Hernia 11:341-346

4. Kurt N, Oncel M, Ozkan Z, Bingul S (2003) Risk and outcome of bowel resection in patients with incarcerated groin hernias: retrospective study. World J Surg 27:741-743

5. Hentati H, Dougaz W, Dziri C (2014) Mesh repair versus nonmesh repair for strangulated inguinal hernia: systematic review with meta-analysis. World J Surg 38:2784-2790

6. Wysocki A, Strzalka M, Migaczewski M, Budzynski P (2014) Short- and long-term outcomes of incarcerated inguinal hernias repaired by Lichtenstein technique. Wideochir Inne Tech Maloinwazyjne 9:196-200

7. Sawayama H, Kanemitsu K, Okuma T et al (2014) Safety of polypropylene mesh for incarcerated groin and obturator hernias: a retrospective study of 110 patients. Hernia 18:399-406

8. Derici H, Unalp HR, Nazli O et al (2010) Prosthetic repair of incarcerated inguinal hernias: is it a reliable method? Langenbecks Arch Surg 395:575-579

9. Sajid MS, Leaver C, Baig MK, Sains P (2012) Systematic review and meta-analysis of the use of lightweight versus heavyweight mesh in open inguinal hernia repair. Br J Surg 99:29-37

10. Elsebae MM, Nasr M, Said M (2008) Tension-free repair versus Bassini technique for strangulated inguinal hernia: a controlled randomized study. Int J Surg 6:302-305

11. Alimoglu O, Kaya B, Okan I et al (2006) Femoral hernia: a review of 83 cases. Hernia 10:70-73

12. Horan TC, Gaynes RP, Martone WJ et al (1992) CDC definitions of nosocomial surgical site infections, 1992: a modification of CDC definitions of surgical wound infections. Infect Control Hosp Epidemiol 13:606-608

13. Tatar C, Tuzun IS, Karsidag T et al (2016) Prosthetic mesh repair for incarcerated inguinal hernia. Balkan Med J 33:434-440

14. Bittner JGt (2016) Incarcerated/strangulated hernia: open or laparoscopic? Adv Surg 50:67-78

15. Ozbagriacik M, Bas G, Basak F et al (2015) Management of strangulated abdominal wall hernias with mesh; early results. North Clin Istanb 2:26-32

16. Dunne JR, Malone DL, Tracy JK, Napolitano LM (2003) Abdominal wall hernias: risk factors for infection and resource utilization. J Surg Res 111:78-84

17. Kulah B, Kulacoglu IH, Oruc MT et al (2001) Presentation and outcome of incarcerated external hernias in adults. Am J Surg 181:101-104

18. Alvarez JA, Baldonedo RF, Bear IG et al (2004) Incarcerated groin hernias in adults: presentation and outcome. Hernia 8:121-126 
19. Atila K, Guler S, Inal A et al (2010) Prosthetic repair of acutely incarcerated groin hernias: a prospective clinical observational cohort study. Langenbecks Arch Surg 395:563-568

20. Hellspong G, Gunnarsson U, Dahlstrand U, Sandblom G (2017) Diabetes as a risk factor in patients undergoing groin hernia surgery. Langenbecks Arch Surg 402:219-225

21. Kaoutzanis C, Leichtle SW, Mouawad NJ et al (2015) Risk factors for postoperative wound infections and prolonged hospitalization after ventral/incisional hernia repair. Hernia 19:113-123

22. Yang L, Wang H, Liang X et al (2015) Bacteria in hernia sac: an important risk fact for surgical site infection after incarcerated hernia repair. Hernia 19:279-283

23. Nilsson H, Nilsson E, Angeras U, Nordin P (2011) Mortality after groin hernia surgery: delay of treatment and cause of death. Hernia 15:301-307

24. Campanelli G, Nicolosi FM, Pettinari D, Avesani EC (2004) Prosthetic repair, intestinal resection, and potentially contaminated areas: safe and feasible? Hernia 8:190-192

25. Bakota B, Kopljar M, Baranovic S et al (2015) Should we abandon regional anesthesia in open inguinal hernia repair in adults? Eur J Med Res 20:76
26. Yang S, Zhang G, Jin C et al (2016) Transabdominal preperitoneal laparoscopic approach for incarcerated inguinal hernia repair: a report of 73 cases. Med (Baltimore) 95:e5686

27. Burcharth $\mathbf{J}$ (2014) The epidemiology and risk factors for recurrence after inguinal hernia surgery. Dan Med J 61:B4846

28. Wysocki A, Kulawik J, Pozniczek M, Strzalka M (2006) Is the Lichtenstein operation of strangulated groin hernia a safe procedure? World J Surg 30:2065-2070

29. Pandey H, Thakur DS, Somashekar U et al (2018) Use of polypropylene mesh in contaminated and dirty strangulated hernias: short-term results. Hernia. https://doi.org/10.1007/s1002 9-018-1811-3

30. Matsuda A, Miyashita M, Matsumoto S et al (2018) Laparoscopic transabdominal preperitoneal repair for strangulated inguinal hernia. Asian J Endosc Surg 11:155-159

31. Ueda J, Nomura T, Sasaki J et al (2012) Prosthetic repair of an incarcerated groin hernia with small intestinal resection. Surg Today 42:359-362

32. Kockerling F, Alam NN, Narang SK et al (2015) Biological meshes for inguinal hernia repair-review of the literature. Front Surg 2:48 